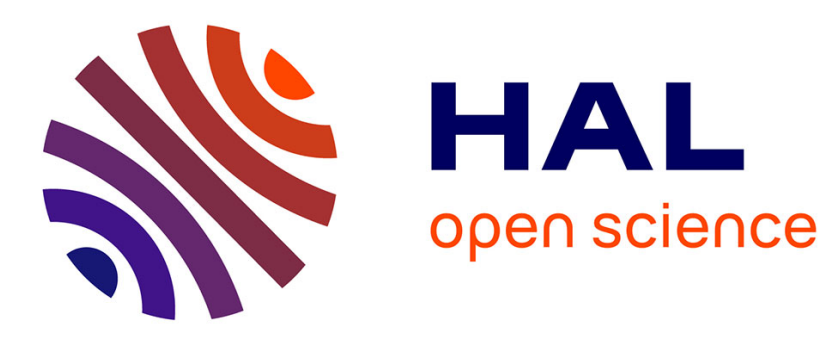

\title{
Genetics of somatic incompatibility in Collybia fusipes
}

Benoit Marçais, O. Caël, C. Delatour

\section{To cite this version:}

Benoit Marçais, O. Caël, C. Delatour. Genetics of somatic incompatibility in Collybia fusipes. Mycological Research, 2000, 104 (3), pp.304-310. 10.1017/S0953756299001069 . hal-02061543

\section{HAL Id: hal-02061543 https://hal.science/hal-02061543}

Submitted on 8 Mar 2019

HAL is a multi-disciplinary open access archive for the deposit and dissemination of scientific research documents, whether they are published or not. The documents may come from teaching and research institutions in France or abroad, or from public or private research centers.
L'archive ouverte pluridisciplinaire HAL, est destinée au dépôt et à la diffusion de documents scientifiques de niveau recherche, publiés ou non, émanant des établissements d'enseignement et de recherche français ou étrangers, des laboratoires publics ou privés. 


\title{
Genetic of somatic incompatibility in Collybia fusipes
}

\author{
Marçais, B., Caël, O., and Delatour, C. \\ Laboratoire de Pathologie Forestière. Institut National de la Recherche \\ Agronomique, 54280 Champenoux, France
}

\begin{abstract}
The genetic of somatic incompatibility in tetrapolar Collybia fusipes was studied using eight dikaryotic isolates collected from the wild and their experimentally derived progeny. Monokaryons from each isolate were all paired with the same unrelated monokaryon and also paired together in all combinations. The somatic compatibility of the two resulting sets of dikaryons was studied. Two different types of somatic incompatible interaction were observed, (1) a lightly pigmented line developing between the two isolates; (2) a heavily pigmented line developing between the two isolates. The dikaryons that had one nuclear type in common and one coming from sibling monokaryons were compatible in $7-27 \%$ of the cases, incompatible with a lightly pigmented interaction in 30-93\% and incompatible with a heavily pigmented interaction in $0-53 \%$. The results suggest that at least three to four loci control the somatic incompatibility in $C$. fusipes, one of them controlling alone the heavily pigmented interaction.
\end{abstract}

\section{Introduction}

Collybia fusipes (Bull. Ex Fr.) Quél. is a pathogenic basidiomycetous fungus that is a common cause of root rot on mature oaks in France (Guillaumin et al, 1985; Département de Santé des Forêts, 1994; Marçais \& Delatour, 1996; Marçais, Caël \& Delatour, 1998a).

Somatic incompatibility (SI), the interaction that usually develops between the mycelia of two unrelated dikaryons whenever they come into contact in wood or on agar medium, has been successfully used to study the structure of the population of many root and wood rotting basidiomycetes (Barrett \& Uscuplic, 1971; Childs, 1963; Holmer, Nitare \& Stenlid, 1994; Kile, 1983; Lewis \& Hansen, 1991; Worral, 1994). We have used it to determine the structure of Collybia fusipes populations in infected stands of oaks (Marçais, Martin \& Delatour, 1998b). Isolates from neighbouring infected oak trees were shown to be incompatible most of the time, while isolates coming from the same root system were compatible in $65 \%$ of the cases. However, we do not know the genetic relationship between compatible isolates. It is often assumed that compatible isolates are members of the same genet (Barrett \& Uscuplic, 1971; Childs, 1963; Holmer et al, 1994; Kile, 1983; Lewis \& Hansen, 1991; 
Worral, 1994), but they were shown to have no genetic relationship in at least one mycorrhizal basidiomycete (Jacobson et al, 1993).

In contrast to the $\mathrm{SI}$ interactions that occur between dikaryons in homobasidiomycetes, those in the ascomycetes takes place between monokaryons where the genetics have been well studied and many genes are involved. Eleven genes, including those at the mating type locus, control SI in Neurospora crassa (Perkins, 1988), at least five in Cryphonectria parasitica (Anagnostakis, 1982), nine in Podospora anserina (Bérgueret, Turcq \& Clavé, 1994) and eight in Aspergillus nidulans (Croft \& Dales, 1984). Two isolates are compatible only if they have the same alleles at each of the genes that control SI.

The genetic control of SI has also been studied recently in several basidiomycetes (Hansen, Stenlid \& Johansson, 1993; Hansen, Stenlid \& Johansson, 1994; Rizzo, Rentmeester \& Burdsall, 1995; Worral, 1997; Guillaumin, 1998). The genetic of $\mathrm{SI}$ in homobasidiomycetes are not well understood. Compared to ascomycetes, fewer genes seems to be involved. For example, just one locus was shown to control SI in Phellinus weirii (Hansen et al, 1994) while 2 closely linked loci control SI in Armillaria ostoyae (Guillaumin, 1998). A low number of loci controlling SI might also explain why sibling dikaryons were shown to be compatible in $40-50 \%$ of the cases in several Armillaria species other than A. ostoyae and in $98 \%$ in Phaeolus schweinitzii (Barrett \& Uscuplic, 1971; Korhonen, 1978; Kile, 1983).

We studied the genetic of $S I$ in $C$. fusipes to be able to better interpret our results on the distribution of vegetative compatibility groups of this fungus in infected stands of oaks (Marçais et al, 1998b). We also wanted to investigate the genetic basis of SI in this fungus, especially that of closely related dikaryons.

\section{Material and Methods}

\section{Basidiome production and isolation of monokaryons}

Eight dikaryotic C. fusipes isolates, C2, C13, C14, C37, C38, C49, C50 and C52, were studied (Table 1). Monokaryotic isolates were obtained from basidiomes produced in vitro using the following procedure: four $1.0 \mathrm{~cm}^{2}$ blocks from a $C$. fusipes culture growing on $2 \%$ malt agar were placed in a $250 \mathrm{ml}$ Erlenmeyer flask containing $10 \mathrm{~g}$ of dry beech sawdust, $20 \mathrm{~g}$ of whole grain brown rice, $0.72 \mathrm{~g}$ of Bacto-peptone and $120 \mathrm{ml}$ of distilled water that had been previously autoclaved at $120^{\circ} \mathrm{C}$ for $20 \mathrm{~min}$. The medium was adapted from Tirro (1991). Four Erlenmeyer flasks were inoculated per isolate, incubated at $23^{\circ} \mathrm{C}$ in the dark for two months and then placed in an incubator with a $16 \mathrm{~h}$ light period under $2500 \mathrm{Lux}$ at $15^{\circ} \mathrm{C}$ and an $8 \mathrm{~h}$ dark period at $10^{\circ} \mathrm{C}$ until the appearance of basidiomes, according to the recommendation of Tirro (personal communication). Basidiomes appeared after 3 6 months. Basidiospores were collected by placing a sterile $18 \mathrm{~mm} \times 18 \mathrm{~mm}$ cover slip overnight under the basidiome. The basidiospores were washed from the cover slip with sterile water and the spore suspension was then plated on five malt agar petri dishes. After an incubation of $7-10$ days at $23^{\circ} \mathrm{C}$, individual germinating basidiospores were located using a binocular microscope, and transferred using a sterile Pasteur pipette to fresh malt agar plates. After an incubation of one week at $23^{\circ} \mathrm{C}$, single hyphae of the developing colonies were transferred to fresh malt agar plates. About thirty monokaryons were isolated from the in vitro produced basidiomes of each isolates. 

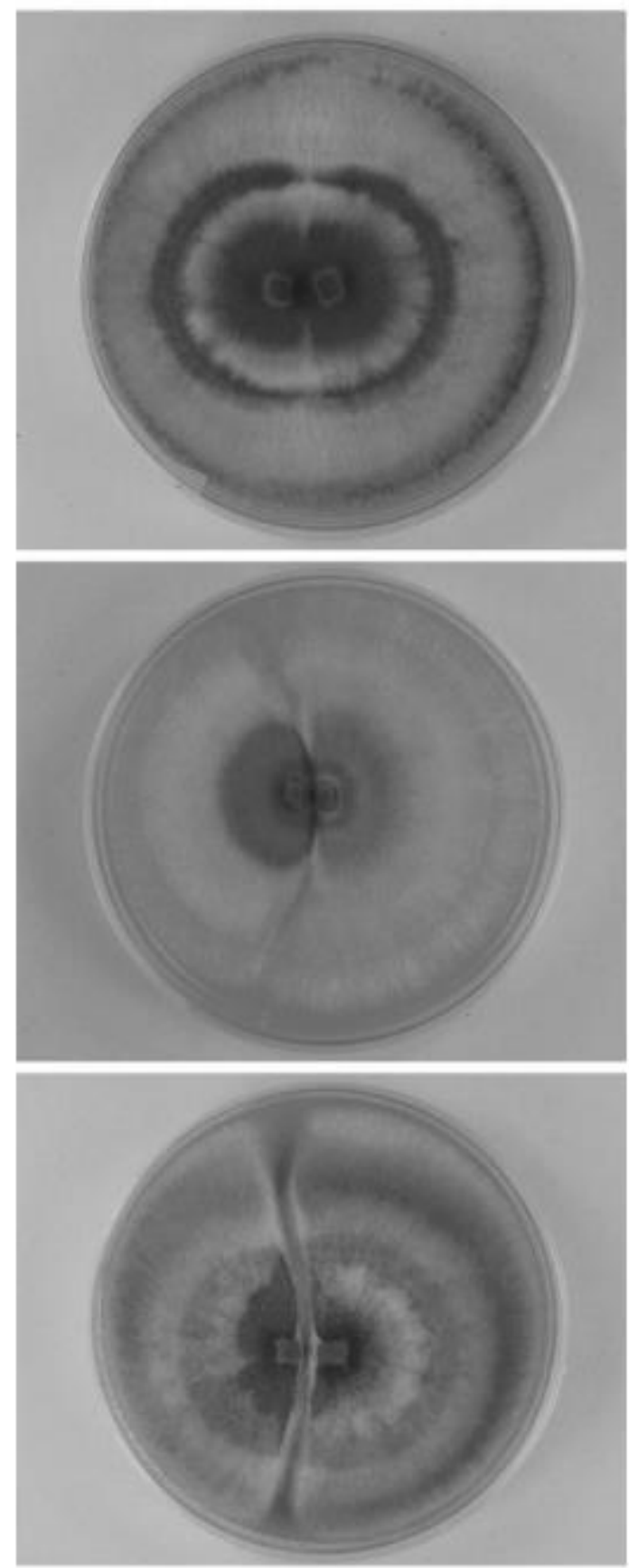

Fig. 1. Somatic interaction between two Collybia fusipes isolates on malt agar medium after 30 days. From the top to the bottom: Compatible interaction rated as 0 (self pairing); Weakly incompatible interaction rated as 1; Strongly incompatible interaction rated as 2.

\section{Production of synthetic dikaryons}

Synthetic dikaryons were produced by pairing monokaryons on malt agar medium at room temperature. Agar blocks about $4 \mathrm{~mm}^{2}$ containing actively growing mycelium from each of two monokaryons were placed in the centre of a plate about $5 \mathrm{~mm}$ apart. One plate was done for each pairing. After an incubation of one month, isolations were made from the area of intermingling hyphae that developed between 
the colonies. After one week, the status, monokaryotic or dikaryotic, of the new strain was assessed. The presence of clamp connections or a change in colony morphology indicated an dikaryotic colony. Dikaryotic colonies turned brown in the older parts, while monokaryotic colonies stayed white. The dikaryons were kept on malt agar medium for further studies.

Two different types of pairings were established for each set of sibling monokaryons. The mating types of fifteen sibling monokaryons were determined by pairing them in all combinations. The dikaryons obtained from those pairings will be referred to as sibling dikaryons. All fifteen sibling monokaryons were also paired to the same unrelated monokaryon. The dikaryons obtained from those pairings therefore all had one nucleus in common and one from sibling monokaryons. They will be referred to as sib-related dikaryons.

\section{Incompatibility tests}

Sib-related dikaryons obtained from the isolates from the wild were paired together in all combinations. Up to fifteen of the sibling dikaryons obtained from these eight progenies were also paired together in all combinations. Two dikaryons were paired by placing $4 \mathrm{~mm}^{2}$ agar blocks containing actively growing mycelium $5 \mathrm{~mm}$ apart on cristomalt medium (15 g Agar, $20 \mathrm{~g}$ cristomalt Difal, 1 I distilled water). Each of the pairings was done only once. The morphology of the interaction was studied following incubation at room temperature for 15,30 and 45 days. Pairings were rated as 0 , no visible reaction; 1 , light pigmentation appearing at 30 days after incubation; 2, heavy pigmentation present 15 days after incubation (Fig. 1). Heavily pigmented interactions will hereafter be referred to as strong incompatibility whereas the lightly pigmented interactions will be referred to as weak incompatibility.

Table 1. Origin of the Collybia fusipes isolates used in this work

\begin{tabular}{|c|c|c|c|}
\hline $\begin{array}{l}\text { Isolat } \\
\text { e }\end{array}$ & $\begin{array}{c}\text { year of } \\
\text { isolatio } \\
\mathrm{n}\end{array}$ & $\begin{array}{l}\text { Host of } \\
\text { isolation }\end{array}$ & Origin and Place of Isolation \\
\hline $\mathrm{C} 2$ & 1983 & Q. robur & infected tissues, Stanesti forest, Romania \\
\hline $\mathrm{C} 13$ & 1982 & Q. robur & infected tissues, Forêt de Tronçais, Allier, France \\
\hline $\mathrm{C} 14$ & 1982 & Q. robur & $\begin{array}{l}\text { infected tissues, Forêt de Mouguerre, Pyrénées Atlantiques, } \\
\text { France }\end{array}$ \\
\hline C37 & 1993 & Q. petreæ & infected tissues, Hackel forest, Saxony-Anhalt, Germany \\
\hline C38 & 1993 & Q. robur & infected tissues, Experimental forest of Kornik, Poland ${ }^{a}$ \\
\hline C49 & 1992 & Q. rubra & infected tissues, Arboretum des Barres, Loiret, France \\
\hline C50 & 1992 & Q. rubra & basidiome, Arboretum d'Amance, Meurthe et Moselle, France \\
\hline C52 & 1992 & Q. robur & basidiome, Forêt de Mersuay, Haute Sâone, France \\
\hline P37.1 & 1996 & - & $\begin{array}{l}\text { result of an in vitro pairing between monokaryons } \mathrm{C}^{3}{ }_{[1]} \text { and } \\
\text { C37 } 5771\end{array}$ \\
\hline P37.2 & 1996 & - & $\begin{array}{l}\text { result of an in vitro pairing between monokaryons } \mathrm{C}{ }_{[1]} \text { and } \\
\mathrm{C} 37_{[51}\end{array}$ \\
\hline P37.3 & 1996 & - & $\begin{array}{l}\text { result of an in vitro pairing between monokaryons } \mathrm{C}_{[14]} \text { and } \\
\text { C37 }\end{array}$ \\
\hline
\end{tabular}




\section{Pairings between sib-related dikaryons and dikaryons obtained from parent monokaryons}

The progenies from three synthetic dikaryons, P37.1, P37.2 and P37.3, were studied (Table 1). These isolates are sibling dikaryons obtained by pairing together monokaryons from the C37 isolate. P37.1 and P37.3 would be homozygous for the hypothesized single locus controlling strong incompatibility, while P37.2 would be homozygous for that locus (see Table 3). Respectively 28, 12 and 17 monokaryons were isolated from basidiomes produced in vitro by P37.1, P37.2 and P37.3. Sibrelated dikaryons were produced by pairing the P37.1 $[\mathrm{n}], \mathrm{P} 37.2_{[n]}$ and $\mathrm{P} 37.3_{[\mathrm{n}]}$ progenies with $\mathrm{C} 50_{[1]}$. The sib-related dikaryons $\mathrm{P} 37.1_{[\mathrm{n}]} \times \mathrm{C} 50_{[1]}, \mathrm{P} 37.2_{[\mathrm{n}]} \times \mathrm{C}_{50} 0_{[1]}$ and $\mathrm{P} 37.3_{[\mathrm{n}]} \times \mathrm{C} 50_{[1]}$ were all paired with $\mathrm{C}_{3} 7_{[1]} \times \mathrm{C} 50_{[1]}$ and $\mathrm{C} 37_{[14]} \times \mathrm{C} 50_{[1]}$.

Table 2. Somatic incompatibility between sib-related dikaryons differing at only one nuclear type

\begin{tabular}{|c|c|c|c|c|c|}
\hline \multirow[t]{2}{*}{$\begin{array}{l}\text { Set of } \\
\text { dikaryons }\end{array}$} & \multirow[t]{2}{*}{$\begin{array}{l}\text { Number of } \\
\text { dikaryons }\end{array}$} & \multirow[t]{2}{*}{$\begin{array}{l}\text { Number of } \\
\text { pairings } b\end{array}$} & \multicolumn{3}{|c|}{$\begin{array}{c}\text { Pairing rated } \\
\text { as (\%) C }\end{array}$} \\
\hline & & & 0 & 1 & 2 \\
\hline $\mathrm{C} 2_{[\mathrm{n}]} \times \mathrm{C} 52_{[1]}^{\mathrm{a}}$ & 15 & 105 & 6.7 & 42.0 & 51.3 \\
\hline $\mathrm{C}^{1} 3_{[\mathrm{n}]} \times \mathrm{C} 50_{[1]}$ & 15 & 105 & 27.6 & 30.5 & 41.9 \\
\hline $\mathrm{C} 14_{[\mathrm{n}]} \times \mathrm{C} 50_{[1]}$ & 15 & 105 & 8.6 & 57.1 & 34.3 \\
\hline $\mathrm{C} 37_{[\mathrm{n}]} \times \mathrm{C} 50_{[1]}$ & 15 & 105 & 12.4 & 34.3 & 53.3 \\
\hline $\mathrm{C} 38_{[\mathrm{n}]} \times \mathrm{C} 49_{[1]}$ & 15 & 105 & 9.5 & 42.9 & 47.6 \\
\hline $\mathrm{C} 49_{[n]} \times \mathrm{C} 38_{[16]}$ & 15 & 105 & 12.4 & 87.6 & 0.0 \\
\hline $\mathrm{C} 50_{[\mathrm{n}]} \times \mathrm{C} 13_{[1]}$ & 15 & 105 & 6.6 & 93.4 & 0.0 \\
\hline $\mathrm{C}^{2}[\mathrm{n}] \times \mathrm{C} 0_{[1]}$ & 15 & 105 & 7.6 & 64.8 & 27.6 \\
\hline \multicolumn{2}{|l|}{$\begin{array}{l}\text { Proportion of the } \\
\text { incompatible pairings (\%) }\end{array}$} & & & 62.9 & 37.1 \\
\hline \multicolumn{5}{|c|}{$\begin{array}{l}\text { a dikaryons obtained by pairing a set of sibling monokaryons, }{ }^{C} 2[n] \text {, to } \\
\text { unique unrelated monokaryon, } C 52[1] \\
\text { b excluding the self pairings }\end{array}$} & a \\
\hline
\end{tabular}

\section{Results}

The weak incompatibility interactions (rating of 1 ) were very heterogeneous. In some pairings, the pigmentation was very light and diffuse and the interaction was difficult to separate from compatibility. In others, a clear pigmented line was present. A useful criterion was the ability of the two colonies to form a common pseudosclerotial plate. After an incubation of one month, C. fusipes colonies commonly form a brown crust that covers part of the colony and extends through the medium to the bottom of the plate, enclosing a portion of the medium. Colonies of compatible dikaryons formed crusts that enclosed the two initial blocks; this was never observed in slightly incompatible dikaryons. The weak incompatibility interactions (rating of 1) were, however, easy to distinguish from the strong incompatibility interactions (rating of 2 ) which were very homogeneous. The line 
between the 2 colonies was more heavily pigmented and developed faster than in weak incompatibility interactions and the gap between the 2 colonies was wider (at least 3-4 mm, Fig. 1). Pairings that were rated as 2 at 15 days were always rated as 2 also at 30 or 45 days while those rated as 0 or 1 at 30 days were never rated as 2 at 45 days.

Table 3. Somatic incompatibility between the sib-related $\mathrm{C} 37_{[\mathrm{n}]} \times \mathrm{C}_{50} 0_{[1]}$ set of dikaryons a

\begin{tabular}{|c|c|c|c|c|c|c|c|c|c|c|c|c|c|c|c|c|c|}
\hline & $\begin{array}{l}\text { Mating } \\
\text { type }\end{array}$ & Group $^{c}$ & 1 & 2 & 3 & 4 & 5 & 6 & 7 & 8 & 9 & 10 & 11 & 12 & 13 & 14 & 15 \\
\hline $1: \mathrm{C} 37_{[11]} \times \mathrm{C} 50_{[11]}$ & $A_{2} B_{2}$ & G1 & $0^{d}$ & & & & & & & & & & & & & & \\
\hline 2: $\mathrm{C} 37_{[3]} \times \mathrm{C}_{[} 0_{[11}$ & $A_{2} B_{2}$ & G1 & 0 & 0 & & & & & & & & & & & & & \\
\hline 3: $\mathrm{C} 37_{[111} \times \mathrm{C} 50_{[11]}$ & $A_{2} B_{1}$ & G1 & 0 & 0 & 0 & & & & & & & & & & & & \\
\hline 4: C37 $\left[151 \times\right.$ X $50_{[11]}$ & $A>B_{1}$ & G1 & 0 & 0 & 0 & 0 & & & & & & & & & & & \\
\hline 5: C37 & $A_{1} B_{2}$ & G1 & 1 & 1 & 1 & 1 & 0 & & & & & & & & & & \\
\hline 6: $\mathrm{C} 37_{\lceil 271} \times \mathrm{C} 50_{[11]}$ & $A_{1} B 1$ & G1 & 1 & 1 & 1 & 1 & 0 & 0 & & & & & & & & & \\
\hline $7: \mathrm{C}_{3} 7_{[4]} \times \mathrm{C} 50_{[1]}$ & $A_{2} B$ ? & G1 & 1 & 1 & 1 & 1 & 1 & 1 & 0 & & & & & & & & \\
\hline 8: $\mathrm{C} 37_{[6]} \times \mathrm{C} 50_{[11}$ & $A_{2} B_{2}$ & G1 & 1 & 1 & 1 & 1 & 1 & 1 & 1 & 0 & & & & & & & \\
\hline 9: $\mathrm{C} 37_{[5]} \times \mathrm{C} 50_{[11]}$ & $A_{1} B_{1}$ & G2 & 2 & 2 & 2 & 2 & 2 & 2 & 2 & 2 & 0 & & & & & & \\
\hline 10: $\mathrm{C} 37_{[7]} \times \mathrm{C} 50_{[11]}$ & $A_{2} B_{2}$ & G2 & 2 & 2 & 2 & 2 & 2 & 2 & 2 & 2 & 0 & 0 & & & & & \\
\hline 11: $\mathrm{C} 37_{[17]} \times \mathrm{C} 0_{[1]}$ & $A_{1} B_{2}$ & G2 & 2 & 2 & 2 & 2 & 2 & 2 & 2 & 2 & 0 & 0 & 0 & & & & \\
\hline 12: $\mathrm{C} 37_{[14]} \times \mathrm{C} 50_{[11}$ & $A_{2} B_{1}$ & G2 & 2 & 2 & 2 & 2 & 2 & 2 & 2 & 2 & 1 & 1 & 1 & 0 & & & \\
\hline 13: $\mathrm{C} 37_{[9]} \times \mathrm{C} 50_{[11]}$ & $A_{1} B_{2}$ & G2 & 2 & 2 & 2 & 2 & 2 & 2 & 2 & 2 & 1 & 1 & 1 & 0 & 0 & & \\
\hline 14: $\mathrm{C} 37_{[\supset 5]} \times \mathrm{C} 50_{[11]}$ & $A>B_{1}$ & G2 & 2 & 2 & 2 & 2 & 2 & 2 & 2 & 2 & 1 & 1 & 1 & 0 & 0 & 0 & \\
\hline $15: \mathrm{C}_{3}{ }_{[8]} \times \mathrm{C} 50_{[1]}$ & $A_{2} B_{1}$ & G2 & 2 & 2 & 2 & 2 & 2 & 2 & 2 & 2 & 1 & 1 & 1 & 1 & 1 & 1 & 0 \\
\hline
\end{tabular}

a Dikaryons obtained by pairing a set of 15 sibling monokaryons from a basidiome of the isolate C37 with a unrelated monokaryon $\mathrm{C} 50_{[1]}$, from isolate $\mathrm{C} 50$

b Mating type of the monokaryon $\mathrm{C} 37_{[n]}$, determined by pairing the set of monokaryons $\mathrm{C} 37[\mathrm{n}]$ together in all combinations

C Monokaryons $\mathrm{C}_{3} 7_{[\mathrm{a}}$ and $\mathrm{C} 37_{[\mathrm{b}]}$ are classified in the same group if dikaryons $\mathrm{C}_{3} 7_{[\mathrm{a}]} \times \mathrm{C}_{50} 0_{[1]}$ and $\mathrm{C} 37_{[\mathrm{b}]} \times \mathrm{C} 50_{[1]}$ have a type 0 or 1 interaction and are classified in a different group if those 2 dikaryons have a type 2 interaction.

d 0 , no detectable interaction; 1 , light pigmentation appearing at 30 days after incubation; 2 , heavy pigmentation present 15 days after incubation

\section{Pairings among sib-related dikaryons}

When sib-related dikaryons were paired together, the proportion of compatible interactions ranged from 6.6 to $27.6 \%$ (Table 2). Weakly incompatible interactions accounted for $30-94 \%$ of the pairings. Strongly incompatible interactions occurred in just 6 out of the 8 sets of sib-related dikaryons and then accounted for $28-53 \%$ of the pairings. Depending on the distribution of lightly and strongly incompatible interactions, sib-related dikaryons from the same progeny fell into two groups that we will call weak incompatibility groups. Table 3 and 4 show the results obtained with the progenies C2 and C37. For progeny C37, the interactions between dikaryons numbered from 1 to 8 (group 1) are all compatible or weakly incompatibles; as are interactions between dikaryons numbered 9 to 15 (group 2). By contrast, interactions between dikaryons of group 1 and dikaryons of group 2 are all strongly incompatible. The same structure is present in the pairings of the C2 progeny (Table 4). The 
mating type genes did not play an obvious role in the somatic incompatibility interaction (Table 3 and 4).

Table 4. Somatic incompatibility between the sib-related $\mathrm{C} 2[\mathrm{n}] \times \mathrm{C}^{2}[1]$ set of dikaryons $a$

\begin{tabular}{|c|c|c|c|c|c|c|c|c|c|c|c|c|c|c|c|c|c|}
\hline & $\begin{array}{l}\text { Mating } \\
\text { type b }\end{array}$ & Group C & 1 & 2 & 3 & 4 & 5 & 6 & 7 & 8 & 9 & 10 & 11 & 12 & 13 & 14 & 15 \\
\hline 1: C2 $[14\rceil \times C 52_{\lceil 11}$ & $A_{4} B_{3}$ & G1 & $0^{d}$ & & & & & & & & & & & & & & \\
\hline 2: C2 $[31$ x C52 111 & $\mathrm{A}_{4} \mathrm{~B}_{4}$ & G1 & 1 & 0 & & & & & & & & & & & & & \\
\hline 3: $C 22_{[1 \mathrm{n}]} \times \mathrm{C} 2_{[1]}$ & $A_{3} B_{4}$ & G1 & 1 & 0 & 0 & & & & & & & & & & & & \\
\hline 4: C2 $[12\rceil \times C_{[11}$ & $\mathrm{A}_{4} \mathrm{~B}_{3}$ & G1 & 1 & 0 & 0 & 0 & & & & & & & & & & & \\
\hline 5: C2 $[5] \times C 52_{\lceil 11}$ & $A_{3} B_{4}$ & G1 & 1 & 1 & 1 & 1 & 0 & & & & & & & & & & \\
\hline $6: C 2[1.3\rceil \times C 52_{[11}$ & $\mathrm{A}_{4} \mathrm{~B}_{4}$ & G1 & 1 & 1 & 1 & 1 & 0 & 0 & & & & & & & & & \\
\hline 7: $\mathrm{C} 2_{[4]} \times \mathrm{C} 52_{[1]}$ & $A_{3} B_{4}$ & G1 & 1 & 1 & 1 & 1 & 1 & 1 & 0 & & & & & & & & \\
\hline 8: $\mathrm{C} 2_{\lceil 61} \times \mathrm{C} 52_{\lceil 11}$ & $\mathrm{A}_{4} \mathrm{~B}_{3}$ & G1 & 1 & 1 & 1 & 1 & 1 & 1 & 0 & 0 & & & & & & & \\
\hline 9: $C 2_{[15]} \times C 52_{[1]}$ & $A_{4} B_{3}$ & G1 & 1 & 1 & 1 & 1 & 1 & 1 & 1 & 1 & 0 & & & & & & \\
\hline 10: $C 2_{[111} \times C 52_{\lceil 11}$ & $A_{3} B_{4}$ & G2 & 2 & 2 & 2 & 2 & 2 & 2 & 2 & 2 & 2 & 0 & & & & & \\
\hline 11: C2 $[$ Г2 $\times$ C52 $[11$ & $\mathrm{A}_{4} \mathrm{~B}_{4}$ & G2 & 2 & 2 & 2 & 2 & 2 & 2 & 2 & 2 & 2 & 1 & 0 & & & & \\
\hline 12: $\mathrm{C} 2_{\lceil 91} \times \mathrm{C} 52_{[11}$ & $\mathrm{A}_{3} \mathrm{~B}_{4}$ & G2 & 2 & 2 & 2 & 2 & 2 & 2 & 2 & 2 & 2 & 1 & 0 & 0 & & & \\
\hline 13: C2[81 x C52[11 & $A_{3} B_{4}$ & G2 & 2 & 2 & 2 & 2 & 2 & 2 & 2 & 2 & 2 & 1 & 1 & 1 & 0 & & \\
\hline 14: $\mathrm{C}_{[11]} \times \mathrm{C} 2_{[1]}$ & $A_{3} B_{4}$ & G2 & 2 & 2 & 2 & 2 & 2 & 2 & 2 & 2 & 2 & 1 & 1 & 1 & 1 & 0 & \\
\hline $15:{ }^{2}[7] \times C 52_{[1]}$ & $A_{3} B_{4}$ & G2 & 2 & 2 & 2 & 2 & 2 & 2 & 2 & 2 & 2 & 1 & 1 & 1 & 1 & 0 & 0 \\
\hline
\end{tabular}

a Dikaryons obtained by pairing a set of 15 sibling monokaryons from a basidiome of the isolate C2 with a unrelated monokaryon C52 [1], from isolate C52

b Mating type of the monokaryon $\mathrm{C}_{[\mathrm{n}]}$, determined by pairing the set of monokaryons $\mathrm{C} 2[\mathrm{n}]$ together in all combinations

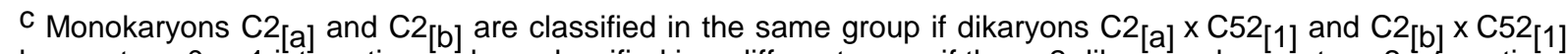
have a type 0 or 1 interaction and are classified in a different group if those 2 dikaryons have a type 2 interaction.

$\mathrm{d}_{0}$, no detectable interaction; 1 , light pigmentation appearing at 30 days after incubation; 2 , heavy pigmentation present 15 days after incubation

\section{Pairings among sibling dikaryons}

In the pairings between sibling monokaryons, $C$. fusipes had a typical tetrapolar mating type system (results not shown). Because the proportions of the 2 alleles were sometimes very unbalanced for both mating type loci, we did not manage to recover 15 sibling dikaryons for each progeny (Table 5). When sibling dikaryons were paired together, the proportion of compatible interactions ranged from 6.7 to $14.7 \%$. Among the incompatible interactions, the proportion of strong incompatibility varied greatly. No strong incompatibility was observed in pairings among sibling dikaryons from C50 and C49, while 35-60\% of the interactions were strong incompatibility for the six other isolates. The sibling dikaryons fell then into 2 or 3 weak incompatibility groups. A representative example is shown in Table 6, for the sibling dikaryons from C37. Group 1 contains dikaryons 1 to 6 , group 2 contains dikaryons 7 to 9 and group 3 dikaryons 10 to 14 . For some progenies, the sibling dikaryons fell in just 2 groups (Table 7). 
Discrepancies were sometime observed in the pairings between siblings or sibrelated dikaryons. For example, in Table 7, dikaryons 4 and 5 are compatible, as are dikaryons 5 and 6 . However, dikaryons 4 and 6 are incompatible.

Table 5. Somatic incompatibility between sibling dikaryons ${ }^{a}$

\begin{tabular}{|c|c|c|c|c|}
\hline \multirow[t]{2}{*}{$\begin{array}{c}\text { Set of } \\
\text { dikaryons }\end{array}$} & \multirow[t]{2}{*}{$\begin{array}{l}\text { Number of } \\
\text { dikaryons }\end{array}$} & \multirow[t]{2}{*}{$\begin{array}{c}\text { Number of } \\
\text { pairing } b\end{array}$} & \multicolumn{2}{|c|}{$\begin{array}{c}\text { Pairing rated } \\
\text { as (\%) C }\end{array}$} \\
\hline & & & 0 & 2 \\
\hline C2 $[$ [n] $\times$ C2 $[\mathrm{n} 1$ & 15 & 105 & 10.541 .9 & 47.6 \\
\hline $\mathrm{C} 13_{[\mathrm{n} 1} \times \mathrm{C} 13_{[\mathrm{n} 1}$ & 10 & 45 & 8.955 .6 & 35.5 \\
\hline $\mathrm{C} 14_{[\mathrm{n}]} \times \mathrm{C} 14_{[\mathrm{n} 1}$ & 15 & 105 & 6.751 .4 & 41.9 \\
\hline C37 $[\mathrm{nl}$ x C37 $[\mathrm{nl}$ & 14 & 91 & 10.021 .0 & 69.0 \\
\hline 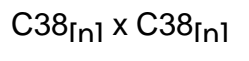 & 6 & 15 & 6.733 .3 & 60.0 \\
\hline $\mathrm{C} 49_{[\mathrm{n} 1} \times \mathrm{C} 49_{[\mathrm{n} 1}$ & 15 & 105 & 6.793 .3 & 0.0 \\
\hline $\mathrm{C} 50_{[\mathrm{n} 1} \times \mathrm{C} 50_{[\mathrm{n} 1}$ & 13 & 75 & 12.088 .0 & 0.0 \\
\hline C52[n] $\times$ C52[n] & 12 & 66 & 14.733 .8 & 51.5 \\
\hline \multicolumn{5}{|c|}{$\begin{array}{l}\text { a dikaryons obtained by pairing together monokaryons } \\
\text { originating from a same basidiome } \\
\mathrm{b} \text { excluding the self pairings }\end{array}$} \\
\hline \multicolumn{5}{|c|}{$\begin{array}{l}\text { C } 0 \text { : no detectable interaction; } 1 \text { : light pigmentation } \\
\text { appearing at } 30 \text { days after incubation; } 2 \text {, heavy } \\
\text { pigmentation present } 15 \text { days after incubation }\end{array}$} \\
\hline
\end{tabular}

Table 6. Somatic incompatibility between the sibling $\mathrm{C} 37[\mathrm{n}]$ set of dikaryons a

\begin{tabular}{|c|c|c|c|c|c|c|c|c|c|c|c|c|c|c|c|}
\hline & $\begin{array}{c}\text { Monokaryons } \\
\text { group b }\end{array}$ & 1 & 2 & 3 & 4 & 5 & 6 & 7 & 8 & 9 & 10 & 11 & 12 & 13 & 14 \\
\hline $1: C 37_{[1]} \times C 37_{[5]}$ & $G_{1} G_{2}$ & $0^{C}$ & & & & & & & & & & & & & \\
\hline 2: C37 $[3] \times \mathrm{C}_{[5]}$ & $G_{1} G_{2}$ & 0 & 0 & & & & & & & & & & & & \\
\hline 3: $\mathrm{C} 37_{[4]} \times \mathrm{C} 37_{[5]}$ & $G_{1} G_{2}$ & 1 & 1 & 0 & & & & & & & & & & & \\
\hline 4: C37 $[8\rceil$ X C37Г211 & $G_{2} G_{1}$ & 1 & 1 & 1 & 0 & & & & & & & & & & \\
\hline 5: C37 $[14\rceil \times C 37_{\lceil 211}$ & $G_{2} G_{1}$ & 1 & 1 & 1 & 0 & 0 & & & & & & & & & \\
\hline 6: C37[211 $\times$ C37Гว:51 & $G_{1} G_{2}$ & 1 & 1 & 1 & 0 & 0 & 0 & & & & & & & & \\
\hline 7: C37 $[6\rceil \times$ C37 & $G_{1} G_{1}$ & 2 & 2 & 2 & 2 & 2 & 2 & 0 & & & & & & & \\
\hline 8: $\mathrm{C} 37_{[11} \times \mathrm{C} 37_{[27\rceil}$ & $\mathrm{G}_{1} \mathrm{G}_{1}$ & 2 & 2 & 2 & 2 & 2 & 2 & 0 & 0 & & & & & & \\
\hline 9: $C 37_{[111]} \times C 3_{[211]}$ & $\mathrm{G}_{1} \mathrm{G}_{1}$ & 2 & 2 & 2 & 2 & 2 & 2 & 1 & 1 & 0 & & & & & \\
\hline 10: C37 $[14] \times C 37_{[17]}$ & $G_{2} G_{2}$ & 2 & 2 & 2 & 2 & 2 & 2 & 2 & 2 & 2 & 0 & & & & \\
\hline 11: C37 $\left[171 \times\right.$ C $37_{\lceil 251}$ & $\mathrm{G}_{2} \mathrm{G}_{2}$ & 2 & 2 & 2 & 2 & 2 & 2 & 2 & 2 & 2 & 0 & 0 & & & \\
\hline 12: C37 $[81 \times$ X C37 $[.91$ & $G_{2} G_{2}$ & 2 & 2 & 2 & 2 & 2 & 2 & 2 & 2 & 2 & 1 & 1 & 0 & & \\
\hline 13: $\mathrm{C} 37_{[9]} \times \mathrm{C} 37_{[141}$ & $G_{2} G_{p}$ & 2 & 2 & 2 & 2 & 2 & 2 & 2 & 2 & 2 & 1 & 1 & 0 & 0 & \\
\hline 14: $\mathrm{C} 37_{[9]} \times \mathrm{C} 37_{[25]}$ & $\mathrm{G}_{2} \mathrm{G}_{2}$ & 2 & 2 & 2 & 2 & 2 & 2 & 2 & 2 & 2 & 1 & 1 & 0 & 0 & 0 \\
\hline
\end{tabular}

a Dikaryons from pairing between 2 monokaryons obtained from a basidiome of the isolate C37

b Monokaryons from isolate $\mathrm{C} 37$ were separated in 2 groups, G1 and G2, according to the results of pairings between the sib-related $\mathrm{C} 37_{[\mathrm{n}]} \times \mathrm{C50}[1]$ set of dikaryons (see table 3 ).

C 0 , no detectable interaction; 1 , light pigmentation appearing at 30 days after incubation; 2 , heavy pigmentation present 15 days after incubation. 


\section{Pairings between sib-related dikaryons and dikaryons obtained from parent monokaryons}

The sib-related dikaryons $\mathrm{P} 37.1_{[\mathrm{n}]} \times \mathrm{C} 50_{[1]}$ were all compatible or weakly incompatible with $\mathrm{C} 37_{[1]} \times \mathrm{C5} 0_{[1]}$ and strongly incompatible with $\mathrm{C} 37_{[14]} \times \mathrm{C50}[1]$ (Table 8). By contrast, the sib-related dikaryons $\mathrm{P} 37.3_{[\mathrm{n}]} \times \mathrm{C} 50_{[1]}$ were all compatible or weakly incompatible with $\mathrm{C} 37_{[14]} \times \mathrm{C} 50_{[1]}$ but strongly incompatible with $\mathrm{C} 37_{[1]} \times \mathrm{C} 50_{[1]}$. Sib-related dikaryons $\mathrm{P} 37_{\text {. }}{ }_{[\mathrm{n}]} \times \mathrm{C} 50_{[1]}$ were of two types: five were compatible or weakly incompatible with $\mathrm{C} 37_{[14]} \times \mathrm{C}_{50}[1]$ and strongly incompatible with $\mathrm{C} 37_{[1]} \times \mathrm{C50}[1]$ and seven were strongly incompatible with $\mathrm{C} 37_{[14]} \times \mathrm{C50}$ [1], but compatible or weakly incompatible with $\mathrm{C} 37_{[1]} \times \mathrm{C} 50_{[1]}$.

Table 7. Somatic incompatibility between the sibling $\mathrm{C}_{2}[\mathrm{n}]$ set of dikaryons ${ }^{\mathrm{a}}$

\begin{tabular}{|c|c|c|c|c|c|c|c|c|c|c|c|c|c|c|c|c|}
\hline & $\begin{array}{c}\text { Monokaryons } \\
\text { group b }\end{array}$ & 1 & 2 & 3 & 4 & 5 & 6 & 7 & 8 & 9 & 10 & 11 & 12 & 13 & 14 & 15 \\
\hline 1: $\mathrm{C} 2_{[1]} \times \mathrm{C} 2_{[6]}$ & $G_{1} G_{2}$ & $0^{c}$ & & & & & & & & & & & & & & \\
\hline 2: $\mathrm{C} 2_{[\supset 1} \times \mathrm{C}_{[\lceil]}$ & $G_{1} G_{2}$ & 0 & 0 & & & & & & & & & & & & & \\
\hline 3: $\mathrm{C2}_{\lceil\lceil]} \times \mathrm{C} 2_{[8]}$ & $G_{1} G_{2}$ & 0 & 0 & 0 & & & & & & & & & & & & \\
\hline 4: $\mathrm{C}_{[11]} \times \mathrm{C}_{[1>1}$ & $G_{>} G_{1}$ & 1 & 1 & 1 & 0 & & & & & & & & & & & \\
\hline 5: C2 $\left[81 \times C 22_{[121}\right.$ & $G_{2} G_{1}$ & 1 & 1 & 1 & 0 & 0 & & & & & & & & & & \\
\hline $6: C_{[11} \times C 2_{[14]}$ & $\mathrm{G}_{1} \mathrm{G}_{2}$ & 1 & 1 & 1 & 1 & 0 & 0 & & & & & & & & & \\
\hline $7: \mathrm{C}_{\lceil 21} \times \mathrm{C} 2_{[14\rceil}$ & $G_{1} G_{2}$ & 1 & 1 & 1 & 1 & 1 & 1 & 0 & & & & & & & & \\
\hline 8: $\mathrm{C} 2_{\lceil 8\rceil} \times \mathrm{C}_{\lceil 141}$ & $G_{2} G_{1}$ & 1 & 1 & 1 & 1 & 1 & 1 & 1 & 0 & & & & & & & \\
\hline 9: $\mathrm{C}_{[111} \times \mathrm{C} 2_{[1.51}$ & $G_{2} G_{1}$ & 1 & 1 & 1 & 1 & 1 & 1 & 1 & 0 & 0 & & & & & & \\
\hline 10: $C 22_{\ulcorner 2\rceil} \times C 2[151$ & $G_{1} G_{2}$ & 1 & 1 & 1 & 1 & 1 & 1 & 1 & 0 & 0 & 0 & & & & & \\
\hline 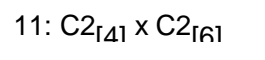 & $G_{1} G_{1}$ & 2 & 2 & 2 & 2 & 2 & 2 & 2 & 2 & 2 & 2 & 0 & & & & \\
\hline 12: $\mathrm{C} 2_{\lceil 6]} \times C 2_{[1.31}$ & $G_{1} G_{1}$ & 2 & 2 & 2 & 2 & 2 & 2 & 2 & 2 & 2 & 2 & 0 & 0 & & & \\
\hline 13: $\mathrm{C}_{\lceil 41} \times \mathrm{C}_{\lceil 12\rceil}$ & $G_{1} G_{1}$ & 2 & 2 & 2 & 2 & 2 & 2 & 2 & 2 & 2 & 2 & 1 & 1 & 0 & & \\
\hline 14: $\mathrm{C} 2_{[12]} \times \mathrm{C} 2_{[1,3]}$ & $G_{1} G_{1}$ & 2 & 2 & 2 & 2 & 2 & 2 & 2 & 2 & 2 & 2 & 1 & 1 & 0 & 0 & \\
\hline $15: \mathrm{C} 2_{[4]} \times \mathrm{C} 2_{[15]}$ & $G_{1} G_{1}$ & 2 & 2 & 2 & 2 & 2 & 2 & 2 & 2 & 2 & 2 & 1 & 1 & 1 & 1 & 0 \\
\hline
\end{tabular}

a Dikaryons from pairing between 2 monokaryons obtained from a basidiome of the isolate $\mathrm{C} 2$

b Monokaryons from isolate C2 were separated into 2 groups, G1 and G2, according to the results of pairings between the sib-related $\mathrm{C}_{[n]}\left[{ } \mathrm{C}^{5}{ }_{[1]}\right.$ set of dikaryons (see table 4).

c 0 , no detectable interaction; 1, light pigmentation appearing at 30 days after incubation; 2, heavy pigmentation present 15 days after incubation

\section{Discussion}

Our results suggest that the strong incompatibility interaction (rated as 2) is controlled by only one locus in Collybia fusipes. In 6 out of the 8 progenies we studied, the sib-related dikaryons fell into 2 well separated weak incompatibility groups. This suggests that 2 alleles of an heterozygous locus segregated. In the other two progenies, C49 and C50, no strong incompatibility was present, which could be explained by homozygosity at that locus. Moreover, as would be expected if strong incompatibility is controlled by one locus, no strong incompatibility was present in pairings between sibling dikaryons from the C49 and C50 isolates, and the sibling dikaryons of the other isolates often fell into 3 weak incompatibility groups. If one deduced which allele each homokaryon carried from the sib-related dikaryon 
pairings (Tables 3 and 4), it can be seen that strong incompatibility occurred only between sibling dikaryons that did not carry exactly the same 2 alleles (Tables 6 and 7). Presence of only 2 weak incompatibility groups in sibling dikaryons from isolate $\mathrm{C} 2$ can be explained by the absence of sibling dikaryons $\mathrm{G}_{2} \mathrm{G}_{2}$ (Table 7).

Table 8. Weak incompatibility groups in the $P 37.1_{[n]} \times C 50_{[1]}$, P37.2[n] $\times$ C50[1] and P37.3[n] $\times$ C50[1] progenies

\begin{tabular}{|c|c|c|c|c|}
\hline & \multicolumn{2}{|c|}{$\mathrm{C} 37_{[1]} \times \mathrm{C} 50_{[1]}$} & \multicolumn{2}{|c|}{$\mathrm{C} 37_{[14]} \times \mathrm{C} 50_{[1]}$} \\
\hline & 0 or 1 & 2 & 0 or 1 & 2 \\
\hline $\mathrm{P} 37.1_{[\mathrm{n}]} \times \mathrm{C} 50_{[1]}$ & 28 & 0 & 0 & 28 \\
\hline $\mathrm{P} 37.2_{[\mathrm{n}]} \times \mathrm{C} 50_{[1]}$ & 5 & 7 & 7 & 5 \\
\hline $\mathrm{P} 37.3_{[\mathrm{n}]} \times \mathrm{C} 50_{[1]}$ & 0 & 17 & 17 & 0 \\
\hline
\end{tabular}

Note: P37.1 results from a pairing between $C 37_{[1]}$ and C37 27$]$, P37.2 from a pairing between $\mathrm{C} 37_{[1]}$ and $\mathrm{C} 37_{[5]}$, and P37.3, from a pairing between $\mathrm{C} 37_{[14}$ and C37 [17]. C37 [1] and C37 [27] are from the first strong incompatibility group in table 3

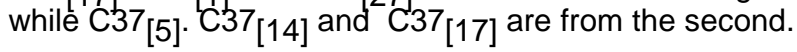

If strong incompatibility is controlled by one locus, we could expect that the alleles segregate in a ratio $1: 1$ and that, in pairing among sib-related dikaryons, when strong incompatibility is present, it represents about $50 \%$ of the pairings. This is not the case for all the progenies, as the proportion of strong incompatibility can be as low as 28 and $35 \%$ in some pairings (Table 2). This might be explained by the small size of the progenies (only 15 monokaryons). Alternatively, strong incompatibility could be the result of an additive response, i.e., interaction being stronger when differences are present at more loci. Though, it is difficult, with this hypothesis, to explain why the sib-related dikaryons were clearly separated in two groups and the sibling dikaryons in three groups. In the case of an additive response, we would expect a progressive increase of the response, with no possibility to separate the sibrelated dikaryons into discrete groups. An additive response is likely for the weak incompatibility as the interactions rated as 1 were very heterogeneous within the sibrelated dikaryons of a progeny and no pattern could be evidenced among those weakly incompatible interactions.

P37.1 and P37.3 are sibling dikaryons both derived from 2 monokaryons that belong to the same weak incompatibility group (C37 ${ }_{[1]}$ and C37 ${ }_{[27]}$ for P37.1; C37 ${ }_{[14]}$ and C37 ${ }_{[17]}$ for P37.3; see Table 3). Thus they would be expected to be homozygous for the hypothesized locus controlling strong incompatibility. As expected in this case, the sib-related $\mathrm{P} 37.1_{[\mathrm{n}]} \times \mathrm{C} 50_{[1]}$ belonged to the same weak incompatible group as $\mathrm{C} 37_{[1]} \times \mathrm{C} 50_{[1]}$ and $\mathrm{P} 37.3_{[n]} \times \mathrm{C} 50_{[1]}$ to the same as $\mathrm{C} 37_{[14]} \times \mathrm{C} 50_{[1]}$. By contrast, P37.2 is derived from sibling monokaryons that belongs to different weak incompatibility groups (C37 ${ }_{[1]}$ and $\mathrm{C} 37_{[5]}$; see Table 3 ). As expected if this dikaryon is heterozygous for a single locus controlling strong incompatibility, five sib-related $\mathrm{P} 37.2_{[n]} \times \mathrm{C} 50_{[1]}$ belonged to the same weak incompatible group as $\mathrm{C} 37_{[1]} \times \mathrm{C} 0_{[1]}$ and seven to the same as $\mathrm{C} 37_{[14]} \times \mathrm{C} 50_{[1]}$. 
It is more difficult to determine the number of loci which are involved in the control of weak incompatibility (rated as 1). If the parent isolate of the sibling nuclear type is heterozygous for only one of the loci controlling SI, then each of the sibling nuclei carry either of the two alleles for that gene and the sib-related dikaryons can belong to just two SI groups. The expected proportion of compatible pairings between the sib-related dikaryons would then be $50 \%$. Similarly, with 2, 3, 4 or 5 heterozygous genes, the expected proportion of compatible pairings between the sib-related dikaryons would be $25 \%, 12.5 \%, 6.3 \%$ or $3.2 \%$. This estimate is based on the assumption that $\mathrm{SI}$ is controlled in this fungus by a genetic system similar to that described in other fungi (Anagnostakis, 1982; Bérgueret et al, 1994; Perkins, 1988; Rizzo et al, 1995). As this proportion varied from 6.7 to $27.4 \%$ among our families of sib-related dikaryons, we could conclude that 2-4 loci were heterozygous in the parental isolates. Assuming strong incompatibility is controlled by one locus, then 1-3 loci controlled weak incompatibility. More loci are probably involved in controlling SI because some genes might have been homozygous in some of the parental isolates. It is also possible that because we studied a relatively small number of monokaryons for each family, we did not recover the two alleles of genes that were heterozygous in the parental isolate.

However, discrepancies were observed in the pairings between siblings and sibrelated dikaryons. This might be because it is sometime difficult to separate very weakly incompatible interactions and compatible interactions. It is also possible that two dikaryons remain compatible if they are different for just one allele at one of the loci controlling weak incompatibility, as has been proposed for other basidiomycetes (Hansen et al, 1993; Worral,1997). Those 2 hypothesis would lead to an overestimate of the number of compatible interactions and so to an under-estimate of the number of loci involved in the weakly pigmented interaction. Alternatively, some genes controlling weak incompatibility might be involved in non-allelic interactions as occurs in $P$. anserina (Bérgueret et al, 1994). In non-allelic interactions, combinations of alleles at two separate loci results in incompatibility.

In another study, we examined the vegetative compatibility of $C$. fusipes isolates collected in two infected oak stands (Marçais et al, 1998b). The morphology of the interactions observed between those unrelated dikaryons was very similar to the one observed among the sib-related and the sibling dikaryons. Weak incompatibility groups were also observed among those wild isolates (9 in one site and 11 in the other). This might be an indication that multiple alleles are present at the locus controlling the strong incompatibility. It is also possible that the strong incompatibility is controlled by several loci that are genetically tightly linked and that we did not detect it in this work because we had only 15 monokaryons in each progeny.

The number of loci controlling $\mathrm{SI}$ in $\mathrm{C}$. fusipes is in a similar range to other studied basidiomycetes. It was found to be three to four in Heterobasidion annosum (Hansen et al, 1993), one in Phellinus weirii (Hansen et al, 1994), two tightly linked in A. ostoyae (Guillaumin, 1998) and at least one in P. gilvus (Rizzo et al, 1995). As in $P$. gilvus, one locus has a major effect on SI (Rizzo et al, 1995). As in $H$. annosum and $P$. gilvus the loci controlling sexual incompatibility do not appear to play a role in SI in C. fusipes (Hansen et al, 1993; Rizzo et al, 1995).

On the basis of our results, vegetative compatibility appears to be a good tool to study the population structure of $C$. fusipes. It is possible with this tool to discriminate between tightly related dikaryons. Indeed, when pairing sibling dikaryons together, 
compatibility was observed in just $7-15 \%$ of the cases. This is also, to our knowledge, the first work reporting a tetrapolar mating type system for $C$. fusipes.

\section{Acknowledgements}

We want to thank F. Kropp for her technical assistance and P. Wargo, P. Frey and $\mathrm{E}$. Hansen for reviewing the manuscript.

\section{Reference}

Anagnostakis, S. L. (1982). Genetic analyses of Endothia parasitica: linkage data for four single genes and three vegetative compatibility types. Genetics 102, 25-28

Barrett, D.K. \& Uscuplic, M. (1971). The field distribution of interacting strains of Polyporus schweinitzii and their origin. New Phytologist 70, 581-598

Bérgueret, J.; Turcq, B., \& Clavé, C. (1994). Vegetative incompatibility in filamentous fungi: het genes begin to talk. Trends in Genetics 10(12), 441-446

Childs, T.W. (1963). Poria weirii root rot. Phytopathology 53, 1124-1127

Croft, J. H.; \& Dales, R. B. G., 1984. Mycelial interactions and mitochondrial inheritance in Aspergillus. In: The ecology and physiology of the fungal mycelium, Ed. D. H. Jennings \& A.D.M., Rayner, pp. 433-450, London/New York: Cambridge Univ. Press

Département de la santé des forêts (France), 1994. La santé des forêts (France) en 1993. Ministère de l'Agriculture et de la pèche (DERF-DSF), 120 pp.

Guillaumin, J. J., Bernard, C., Delatour, C. \& Belgrand, M. (1985). Contribution à l'étude du dépérissement du chêne : pathologie racinaire en forêt de Tronçais. Annales des Sciences Forestières 42, 1-22

Guillaumin, J. J., 1998. Genetic control of somatic incompatibility in Armillaria ostoyae - a preliminary analysis. Pages 31-38, In Proceedings of the ninth International Conference on Root and Butt Rots of Forest Trees (ed. C. Delatour, J.J. Guillaumin, B. LungEscarmant and B. Marçais), Colloques de I'INRA n 89, France.

Hansen, E. M., Stenlid, J. \& Johansson, M. (1993). Genetic control of somatic incompatibility in the root-rotting basidiomycete Heterobasidion annosum. Mycological Research 97, $1229-1233$

Hansen, E.M., Stenlid, J. \& Johansson, M. (1994). Somatic incompatibility in Heterobasidion annosum and Phellinus weirii. pp 323-333. In: Proceedings of the eighth IUFRO root and butt rot conference. Eds. M. Johansson, and J. Stenlid. Swedish University of Agricultural Sciences, Uppsala.

Holmer, L., Nitare, L. \& Stenlid, J. (1994). Population structure and decay pattern of Phellinus tremulae in Populus tremula as determined by somatic incompatibility. Canadian Journal of Botany 72, 1391-1396

Jacobson, K. M., Miller, O. K. \& Turner, B. J. (1993). Randomly amplified polymorphic DNA markers are superior to somatic incompatibility tests for discriminating genotypes in natural populations of the ectomycorrhizal fungus Suillus granulatus. Proceedings of the National Academy of Science of USA 90, 9459-9163

Kile, G. A. (1983). Identification of genotypes and clonal development of Armillaria luteobubalina Watling and Kile in Eucalypt forest. Australian Journal of Botany 31, 657671 
Korhonen, K. (1978). Interfertility and clonal size in the Armillaria mellea complex. Karstenia 18, 31-42

Lewis, K. J., \& Hansen, E. M. (1991). Vegetative compatibility groups and protein electrophoresis indicate a role for basidiospores in spread of Inonotus tomentosus in spruce forests of British Columbia. Canadian journal of Botany 69, 1756-1763

Marçais, B. \& Delatour, C., 1998. Inoculation of Oak (Quercus robur and Q. rubra) with Collybia fusipes. Plant disease 80, 1391-1394

Marçais, B., Caël, C., \& Delatour, C., 1998a. Investigation on the distribution and impact of Collybia fusipes in oak forest. Pages 215-222, In Proceedings of the ninth International Conference on Root and Butt Rots of Forest Trees (ed. C. Delatour, J.J. Guillaumin, B. Lung-Escarmant and B. Marçais), Colloques de I'INRA n 89, France.

Marçais, B., Martin, F., \& Delatour, C., 1998b. Structure of Collybia fusipes population in two infected oak stands. Mycol. Res. 102, 361-367.

Perkins, D. D. (1988). Main features of vegetative incompatibility in Neurospora. Fungal Genetics Newsletter 35, 44-46

Rizzo, D. M., Rentmeester, R. M., \& Burdsall, H. H. (1995). Sexuality and somatic incompatibility in Phellinus gilvus. Mycologia 87, 805-820

Tirro, A. (1991). Tecnica per la producione in vitro dei carpophori di Armillaria. Micologia italiana 20, 73-77

Worrall, J.J. (1994). Population structure of Armillaria species in several forest types. Mycologia 86, 401-407

Worral, J.J. (1997). Somatic incompatibility in basidiomycetes. Mycologia 89, 24-36 